\title{
Comparison and Performance Analysis of App and Web-based One-stop Attendance Management
}

\author{
Sun YuXiang ${ }^{1}$, YoungWook $\mathrm{Cha}^{1}$ and ChoonHee Kim ${ }^{2}$ \\ ${ }^{1}$ Department of Computer Engineering, Andong National University, Korea \\ ${ }^{2}$ Department of Electronic \& Information Communication Engineering, Daegu \\ Cyber University, Korea \\ ywcha@andong.ac.kr
}

\begin{abstract}
This paper presents the structure of an electronic attendance system composed of OAA (One-stop Attendance and Absence)-server and client. We compare simultaneous check, fraudulent attendance, prevention of leaving the classroom, and extra equipment according to check methods, and also present a method of app and web-based attendance management. NFC and BLE-based app and location-based web for attendance management were applied to classrooms. Issues with electronic attendance management and resolution methods were also presented. RESTful web service was defined for attendance management and performance analysis was conducted according to thread numbers of OAA-server and parser types of XML and JSON.
\end{abstract}

Keywords: attendance management; RESTful web service; check-in time; OAAS

\section{Introduction}

Paper-based attendance management has had issues with wasting time on additional efforts required in compiling the logs and manufacturing the attendance $\log$. In order to improve the issues of paper-based attendance management systems, measures such as RFID tags, facial feature recognition, and fingerprint recognition have been developed [14]. But these measures require additional cost through extra equipment and management issues, such as fraudulent attendance by someone else. Smart phones have also become more universal and more than $96.5 \%$ of university students in Korea use smart phones today.

This paper presents the structure of an electronic attendance system composed of OAA (One-stop Attendance and Absence)-server and client. We compare simultaneous check, fraudulent attendance, prevention of leaving the classroom, and extra equipment according to check methods, and also present a method of app and web-based attendance management. By developing an OAA system (OAAS) of lab model, NFC [5] and BLE [6]-based app and location-based [7] web were applied to classrooms. Issues with electronic attendance management and resolution methods were also presented. For expandability, RESTful web service [8] was defined for attendance management between the various smart devices and OAA-server, and performance analysis was conducted according to the thread numbers of web server and parser types of XML and JSON [9]. The analysis result is planned to be used for performance parameters for the design and implementation of electronic attendance system. 


\section{Related Research}

\subsection{Electronic Attendance Management}

Cho [1] suggested a prototype for an attendance management system using NFC (Near Field Communication) and verified whether it can be applied in various education institutions. Through a student's smart phone, the tag information is transmitted to the server, so the SOAP method was used for data transmission and reception. Shi [2] conducted performance analysis and assessed the possibility of actual applications of WiFi-Attendance, which is an automatic attendance checking system using the WiFi signal based on the already established wireless Internet infrastructure and the student's smart phone. In [3], a verification number-based attendance management system was presented to prevent fraudulent attendance done by someone else. The program for the instructor randomly generates verification numbers and presents them on the screen, while the students use their smart phones to re-transmit their seat number and verification number to the program. Lee [4] checks the identity of the attendee by using ID cards with bar codes and verifies the face as well by using the camera on the PC.

\subsection{RESTful Web Service}

SOAP (Simple Object Access Protocol)-based web service has been developed by strict standards of W3C and well-established infrastructure for the programs that use and provide services. RESTful web service can access resources through various expressions (XML, JSON, HTML, images etc.) via only Internet's basic standards [8]. Because of this simplicity and convenience, the information resources from Naver, Daum, Google, Amazon or Yahoo are increasingly opening up via RESTful web service-based API. XML (eXtensible Markup Language) is a standardized grammar and therefore no issues occur between heterogeneous systems in data transmission. Since JSON (JavaScript Object Notation), unlike XML, is an arrangement of letters rather than an exchange method of tags, it is more efficient in analyzing and generating phrases [9]. Oh [10] suggests that the conversion of data exchange methods improved to boost the compatibility of XML and JSON, and shows that the performance can be enhanced through conversion to JSON.

\subsection{Location Information, NFC and BLE}

GPS has high accuracy, but cannot be used to determine the location indoors. In [11], a web app is presented where path discovery is possible on mobile devices using HTML5 with added location functions and mobile communication networkbased location technology, and is converted into a hybrid app through PhoneGap. The accuracy of location varies greatly depending on the cell size and measurement method. Chum [12] describes a WPS (Wireless Positioning System) that selects the user's location from a WiFi AP.

NFC, which is a contact-less wireless communication technology, is installed onto the smart phone for both tagging and reading, and allows two-way communication with little electricity [5]. By merging with smart phones, it is applied in various fields such as electronic attendance [1] and ensures that someone has returned home safely. The beacon, which is based on Bluetooth 4.0 or BLE (Bluetooth Low Energy), is an interior location measurement technology using radio signals as its basis. It can communicate with devices within a maximum of $70 \mathrm{~m}$ radius and is accurate enough to distinguish differences of $5 \sim 10 \mathrm{~cm}$. It also uses very little electricity and is thus fit for implementing the IOT [6]. 


\section{Attendance Check Methods and OAAS Implementation}

\subsection{Comparison of Attendance Check Methods}

In order to identify the applicability of electronic attendance and issues related to its application, a lab model of OAAS was developed, which consisted of the OAAserver and client. OAAS uses only the functions of a smart device to manage attendance electronically, including simultaneous checking, prevention of fraudulent attendance, and leaving classes early after checking in. The OAA-client operating on smart device works as an app through NFC tags, BLE beacons, QR codes and verification numbers. It also operates through a mobile web that uses location information (WiFi AP or cell tower). For Android phones that do not support NFC/BLE or iPhones, a web-based attendance management system was developed using dynamic verification numbers and location information. Table 1 shows simultaneous check, fraudulent attendance, and prevention of leaving the classroom, and extra equipment according to check methods.

Table 1. Comparison of Attendance Check Methods

\begin{tabular}{|c|c|c|c|c|c|}
\hline OAAS & $\begin{array}{c}\text { Check } \\
\text { Method }\end{array}$ & $\begin{array}{c}\text { Extra } \\
\text { Equipment } \\
\end{array}$ & $\begin{array}{c}\begin{array}{c}\text { Concurrent } \\
\text { Check }\end{array} \\
\end{array}$ & $\begin{array}{c}\text { Proxy } \\
\text { Attendance } \\
\end{array}$ & $\begin{array}{c}\text { Prevention of } \\
\text { Movement }\end{array}$ \\
\hline $\mathrm{O}$ & QR code & no & support & difficulty $^{\mathrm{d}}$ & easiness \\
\hline $\mathrm{O}$ & NFC tag ${ }^{\mathrm{a}}$ & $1-2$ tags & waiting line $\mathrm{e}^{\mathrm{c}}$ & difficulty $^{\mathrm{d}}$ & difficulty $^{\mathrm{f}}$ \\
\hline $\mathrm{O}$ & BLE beacon & $1-2$ beacons & support & difficulty $^{\mathrm{d}}$ & easiness \\
\hline $\mathrm{O}$ & Password/app & no & support & difficulty $^{\mathrm{d}}$ & easiness \\
\hline $\mathrm{O}$ & Password/web ${ }^{b}$ & no $^{b}$ & support & difficulty $^{\mathrm{e}}$ & easiness \\
\hline $\mathrm{X}$ & RFID tag $^{\mathrm{a}}$ & many tags/a reader & waiting line $^{\mathrm{c}}$ & easiness & difficulty $^{\mathrm{f}}$ \\
\hline
\end{tabular}

${ }^{\mathrm{a}}$ It is posted on the entrance of classroom.

${ }^{\mathrm{b}}$ It will utilize WiFi AP and cell tower installed in the campus.

${ }^{\mathrm{c}}$ There is waiting line in front of RFID reader or check board with NFC tag.

${ }^{\mathrm{d}}$ Fraudulent attendance is difficult by making use of smart phone's number.

${ }^{\mathrm{e}}$ Fraudulent attendance is difficult by checking dynamic password, location information and IP address of smart device.

${ }^{\mathrm{f}}$ It takes long time because students have to move to the entrance of classroom to check attendance.

\subsection{OAAS Implementation}

Figure 1 shows the structure of OAAS consisting of OAA-client and OAA-server. For the implementation of WGM (Web server and Gui Module) in the OAA-server, the web container Apache-Tomcat and reference implementation Jersey of JAX-RX were used. SAM (Smart Attendance and absence Module) carries out attendance checking, statistics, class management, classroom management, grade management and bulletin board function. PostgreSQL D/B was used to store related information. AVM (Authentication and Validation Module) verifies the validity of the course information and classroom information received from the client, as well as the verification of the student. It also dynamically generates $Q R$ codes or verification numbers. The PHM (Peripheral Handler Module) that is operated in the OAA-client of a smart device carries out the recognition of NFC tag, BLE beacon or QR code in case of attendance check. WSM (Web service for Smart Module) carries out the RESTful web service handler function in order to exchange information on attendance with the OAA-server. The CGM (Coordinator and Gui Module) provides a user interface and serves as a coordinator between PHM and WSM module. 


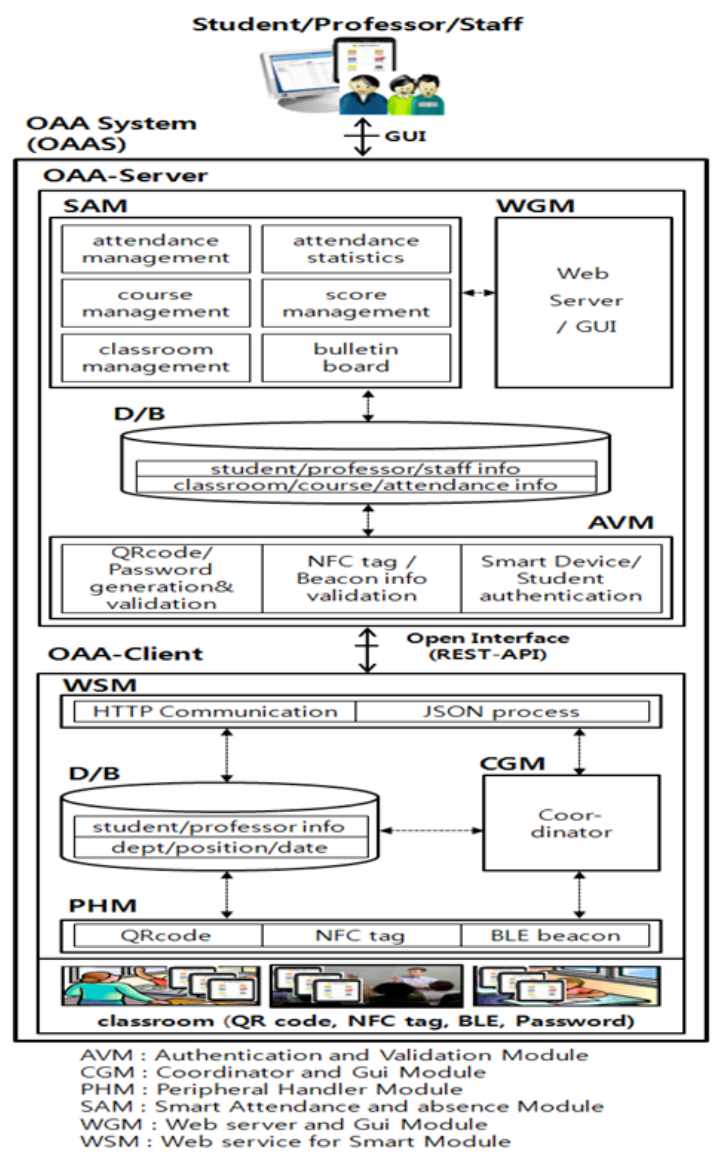

Figure 1. Configuration of OAAS

\section{App and Web-based Attendance Management}

\subsection{App-based Attendance Management}

As seen in (1) of Figure 2, when the app is first operated, the client automatically extracts the phone number of the smart device and requests a login. The server then verifies and approves access. Since it uses phone numbers, only will the fraudulent attending student go unnoticed when he/she has the smart device of the student who is supposed to be there. The client saves the information on student ID, name, and department onto the $\mathrm{D} / \mathrm{B}$ of the smart device and uses the saved information for attendance check. In BLE beacon-based attendance management, Bluetooth authority for a smart device is set and the smart device recognizes the classroom information that is generated by the beacon in the classroom, which is then sent to the server. If it is NFC tag-based, NFC Read/Write mode is set and information (classroom number) from the tag posted on the entrance of the classroom is then recognized and sent to the server.

QR code or verification number-based attendance management is offered for smart devices that do not support NFC or BLE. QR code or verification number is automatically generated by the server and projected onto the screen of the classroom. The smart device sends the class information included in the QR code or the verification number to the server. A smart device exchanges information with OAA-server through JSON-based REST-API with HTTP POST type for login and PUT type for attendance check. Attendance query is done by information exchange through HTML-based REST-API with GET request type using course and student information [8]. 


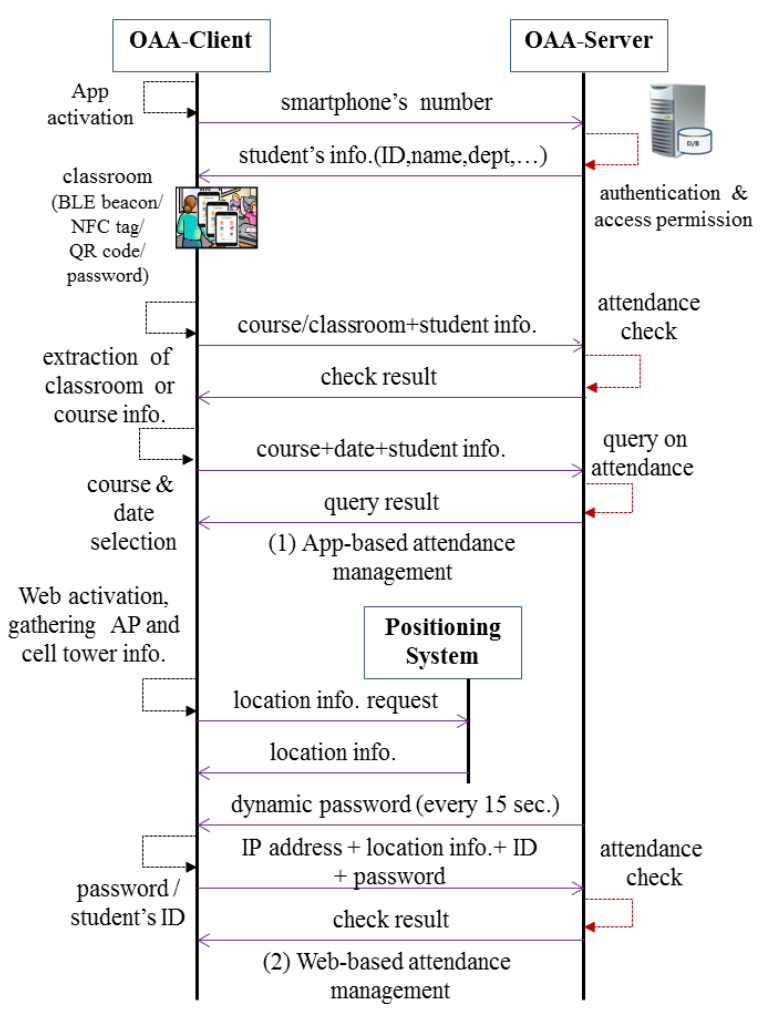

Figure 2. Information Flow of One-stop Attendance Management

\subsection{Web-based Attendance Management}

As can be seen in (2) of Figure 2, when attendance management is operated on the web, the smart device collects neighboring information on the WiFi AP or base station. Using the GeoLocation-API of HTML5 [7], location information (latitude and longitude coordinates, address) is taken from Google's Positioning System. Verification numbers are generated at certain intervals and projected onto the screen in the classroom. The IP address of the smart device, the location information, the student ID number, and valid verification number keyed in by the student are sent to the OAA-server. The server judges the validity of the transmitted information and returns the attendance checking result into the response message. Using location information, it checks whether the student is in the classroom. Fraudulent check through a single smart device can be identified through the inspection of IP address redundancy.

\subsection{Test of One-stop Attendance Management}

4.3.1. Test of App-based Attendance Management: In a classroom with 50 students, the NFC tag and BLE beacon were installed to experiment with the attendance management. BLE is supported only for smart devices with Android version 4.3 (Jelly Bean) or higher so some students with lower versions checked attendance after performing version updates. NFC features are not supported for Apple iOS, and even for Android version 2.3 (Gingerbread) or higher, there are models that do not support NFC such as Galaxy Grand Max. For BLE, the Bluetooth admin authority of Android Manifest is configured so that an automatic pop-up window would be sent out in case of Bluetooth off. For NFC with no manifest authority, if NFC is turned off, the user is sent to the setting screen so that NFC mode could be turned on manually. Most students preferred a BEL-based attendance check-in that didn't require bringing the smart device closer to the NFC tag. 
4.3.2. Test of Web-based Attendance Management: The experiment for web-based attendance management was done in classrooms A and B with 80 and 50 students, respectively. In the first experiment, about $20 \%$ of the students didn't see the attendance management system work. The most frequent cause of this was because the setting for providing location information on the smart device was not done appropriately. The second reason was that the use of GEOLocation API [7] was limited depending on the browser type and version. The third reason was that when base station information was used to collect location information, there was an error with the actual location information. Table 4 shows resolutions for these issues.

Table 2. Solutions and Issues for Web based Attendance Management

\begin{tabular}{|l|l|}
\hline \multicolumn{1}{|c|}{ Issue } & \multicolumn{1}{c|}{ Solution } \\
\hline $\begin{array}{l}\text { No configuration for providing } \\
\text { location information }\end{array}$ & $\begin{array}{l}\text { Configuration guideline for providing location information of } \\
\text { Apple and Android phones }\end{array}$ \\
\hline \multirow{4}{*}{ Web browser's support for } & Installation and utilization of web browsers supporting \\
GEOLocation API & GEOLocation API \\
& - Mozilla Firefox (ver. 3.5+) \\
& - Apple Safari (ver. 5.0+) \\
& - Google Chrome (ver. 5.0+) \\
& - Opera (ver. 16.0+) \\
& - Microsoft Internet Explorer (ver. 9.0+) \\
\hline \multirow{2}{*}{ Location information error } & Utilization of more accurate WiFi AP information instead of \\
& cell tower information \\
\hline
\end{tabular}

As can be seen in Table 2, once issues are resolved in the secondary experiment, most students were able to successfully check in for attendance. To use T-WiFi by SK, which is installed on campus, those who subscribe to KT or LGU+ need to input additional personal data and go through verification. Through this additional verification step, issues with accessing WiFi AP can be resolved.

4.3.3. Comparison between App-based Management and Web-based Management: NFC tags or BLE beacons are installed in the classroom; therefore location information is not additionally required from users in app-based attendance management. Only by setting NFC or Bluetooth can attendance check-in be easily done. In web-based attendance check-in that uses dynamic verification numbers, location information was required to prevent fraudulent attendance; therefore, additional settings were required compared to app-based check-in. The time it takes for traditional attendance logs on paper increases with the number of students, but web or app-based attendance management can be completed within one minute regardless of the number of students.

\section{RESTful Web Service for One-stop Attendance Management}

\subsection{RESTful Web Service Definition for OAA}

For expandability of attendance management, we have defined RESTful web service-based ROAA (RESTful web service for OAA)-API between the OAA-server and various types of smart devices. The information format in ROAA-API is categorized into management object, group and table [13]. Groups represent objects needing simultaneous management, while tables are a series of entries consisting of management objects. ROAA-API uses the request types of HTTP that are GET, POST, PUT, and DELETE, and applies URL rules defined in [14]. In the URL of the request message, "OAA-server address/information type (table, group, and object)/information name" are all commonly included. The most representative request types and URLs of ROAA-API are as shown in Table 3. 
Table 3. ROAA-API's Request Types, URLs, Functions and Status Codes

\begin{tabular}{|c|c|c|c|c|}
\hline \multirow{2}{*}{$\begin{array}{l}\text { request } \\
\text { type }\end{array}$} & \multirow{2}{*}{ URL (http://OAA-server address) } & \multirow{2}{*}{ function } & \multicolumn{2}{|c|}{ status code } \\
\hline & & & ok & error \\
\hline POST & /group/login & authentication & 201 & 400 \\
\hline PUT & /group/attendanceCheck & attendance check & 200 & 400 \\
\hline GET & $\begin{array}{l}\text { /table/attendance/entry/indexes/userId/ }\{\text { userId }\} / \text { cou } \\
\mathrm{rseId} /\{\text { courseId }\}\end{array}$ & query on attendance & 200 & 400 \\
\hline $\begin{array}{c}\text { POST } \\
\text { PUT } \\
\text { DELETE }\end{array}$ & /group/attendanceObjection & objection management & $\begin{array}{l}201 \\
200 \\
204\end{array}$ & 400 \\
\hline GET & /table/objection/entry/indexes/userId/ $\{$ userId $\}$ & objection query & 200 & 400 \\
\hline GET & $/$ table/course/entry/indexes/deptId/ $\{$ deptId $\}$ & $\begin{array}{l}\text { query on courses for a } \\
\text { specific department }\end{array}$ & 200 & 400 \\
\hline $\begin{array}{c}\text { POST } \\
\text { PUT } \\
\text { DELETE }\end{array}$ & /group/courseMakeup & makeup management & $\begin{array}{l}201 \\
200 \\
204\end{array}$ & 400 \\
\hline GET & $\begin{array}{l}\text { /table/classroom/entry/indexes/startTime\&endTime } \\
\text { /\{startTime\&endTime\} }\end{array}$ & $\begin{array}{l}\text { query on classrooms for } \\
\text { makeup class }\end{array}$ & 200 & 400 \\
\hline $\begin{array}{c}\text { POST } \\
\text { PUT } \\
\text { DELETE }\end{array}$ & $\begin{array}{l}\text { /table/classroom/entry/indexes/classroom\&time } /\{\mathrm{cl} \\
\text { assroom\&time }\}\end{array}$ & $\begin{array}{l}\text { classrooms management } \\
\text { for makeup class }\end{array}$ & $\begin{array}{l}201 \\
200 \\
204\end{array}$ & 400 \\
\hline GET & $\begin{array}{l}\text { /table/classroom/entry/indexes/startTime\&endTime } \\
\text { /\{startTime\&endTime\} }\end{array}$ & $\begin{array}{l}\text { query on classrooms for } \\
\text { makeup class }\end{array}$ & 200 & 400 \\
\hline GET & $\begin{array}{l}\text { /table/attendancerate/entry/indexes/course\&stuID/\{ } \\
\text { course\&StuID }\}\end{array}$ & query on attendance rate & 200 & 400 \\
\hline
\end{tabular}

ROAA-API for attendance management uses HTTP PUT type and URL in the form of "group/attendanceCheck". The request message includes attendance method (QR/password/NFC/BLE), check-in order (primary/secondary), student ID number, course code, classroom code, and location information of the smart device. Information on the course, the check-in time, and etc. are included in the response message. The JSON and XML-based ROAA-APIs are as seen in Table 4.

Table 4. ROAA-API for Attendance Check

\begin{tabular}{|c|c|c|}
\hline & JSON & XML \\
\hline $\begin{array}{l}\mathrm{r} \\
\mathrm{e} \\
\mathrm{q} \\
\mathrm{u} \\
\mathrm{e} \\
\mathrm{s} \\
\mathrm{t}\end{array}$ & $\begin{array}{l}\text { \{"oaaCheckGroup": }\{ \\
\text { "chkMethod", } \\
\text { "chkOrder", } \\
\text { "stuID", } \\
\text { "courseID", } \\
\text { "classroom", } \\
\text { "random", } \\
\text { "location"\} } \\
\text { \} }\end{array}$ & $\begin{array}{l}\text { <oaaCheckGroup> } \\
\text { <chkMethod/> } \\
\text { <chkOrder/> } \\
\text { <stuID/> } \\
\text { <courseID/> } \\
\text { <classroom/> } \\
\text { <random/> } \\
\text { <location/> } \\
\text { </oaaCheckGroup> }\end{array}$ \\
\hline $\begin{array}{l}\mathrm{r} \\
\mathrm{e} \\
\mathrm{s} \\
\mathrm{p} \\
\mathrm{o} \\
\mathrm{n} \\
\mathrm{s} \\
\mathrm{e}\end{array}$ & $\begin{array}{l}\text { \{"oaaCheckResGroup": }\{ \\
\text { "year", } \\
\text { "term", } \\
\text { "courseID", } \\
\text { "classroom", } \\
\text { "date", } \\
\text { "lectureTime", } \\
\text { "chkMethod", } \\
\text { "stuID", } \\
\text { "userName", } \\
\text { "time"\} } \\
\text { \} }\end{array}$ & $\begin{array}{l}\text { <oaaCheckResGroup> } \\
\text { <year/> } \\
\text { <term/> } \\
\text { <courseID/> } \\
\text { <classroom/> } \\
\text { <date/> } \\
\text { <lectureTime/> } \\
\text { <chkMethod/> } \\
\text { <stuID/> } \\
\text { <userName/> } \\
\text { <time/> } \\
\text { </oaaCheckResGroup> }\end{array}$ \\
\hline
\end{tabular}




\subsection{Performance Analysis of ROAA-API}

Performance analysis of RESTful web service (ROAA-API) for attendance management was conducted according to XML and JSON parser types, and OAAserver's thread numbers. Only the processing time at the OAA-server was measured, excluding network delay and OAA-client overhead. The processing time is defined as the time it takes between the ROAA-API response message being sent and the request message being received. For performance analysis, 5 OAA-client simulators and an OAA-server of lab model were used. With Apache JMeter [15], a simulator for OAA-client was established. OAA-server and client operate with a 4GB memory capacity and $3.4 \mathrm{GHz}(4$ core $) \mathrm{CPU}$, and run on Windows 7 for 64 bit.

5.2.1. Attendance Check-in Time: Figure 3 shows the check-in time of the OAA server, depending on the parser's type and the arrival rate of the check-in request message. The number of threads was set at 250 , which is the basic configuration for Apache Tomcat. The rate of message arrival is a variant correlated with the number of students requesting attendance check-in at the same time. It was assumed that 10,000 students would be making requests for check-in at the same time over the course of 30 seconds. The message arrival rate was measured up to 300 .

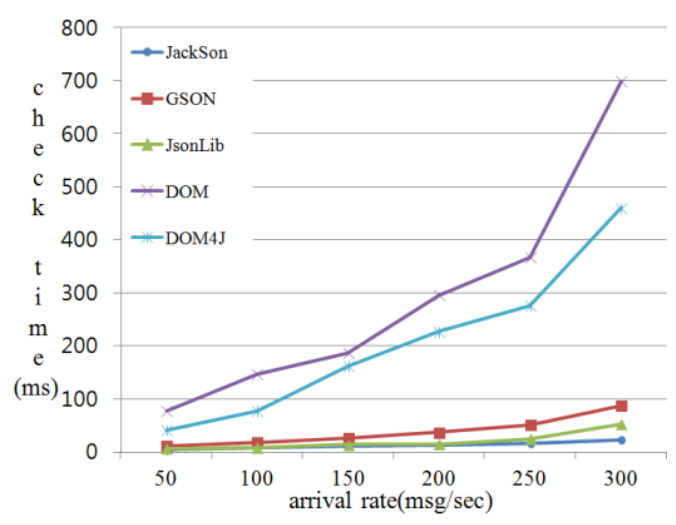

Figure 3. Check-in Time According to Parser Types of JSON and XML

The JSON-based ROAA-API, compared to the XML-based ROAA-API, had a better processing time. Among the JSON parsers JackSon, and among the XML parsers DOM4J showed great performance. When the message arrival rate was 250 , check-in time for JackSon compared to JsonLib was lower by $37.45 \%$, and compared to DOM4J it was lower by $94.48 \%$. Since JSON uses a minimum amount of information, it can be expressed with only about $60 \%$ of what XML would need and is more efficient in analyzing or generating phrases [9].

Figure 4 displays the attendance check-in time of JackSon parser-based API in accordance with Apache Tomcat thread count at 150, 250 and 350, as well as the request message arrival rate. When the number of threads is 250 and the arrival rate is 300 , the processing time decreases by $20 \%$ compared to when the number of threads is 150 , and increases by $14.4 \%$ compared to when the number of threads is 350 . 


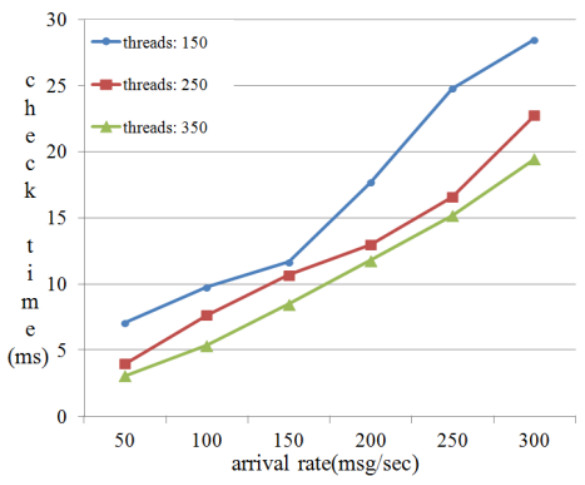

Figure 4. Check-in Time According to the Number of Threads

5.2.2. Attendance Query Time: Figure 5 shows OAA-server's attendance query time in accordance with Apache Tomcat thread count at 150, 250, and 350. When the number of threads was 350 and the arrival rate was 300 , the attendance query time decreased by $44.4 \%$ compared to when the number of threads was 150 , and decreased by $31.7 \%$ compared to when the number of threads was 250 .

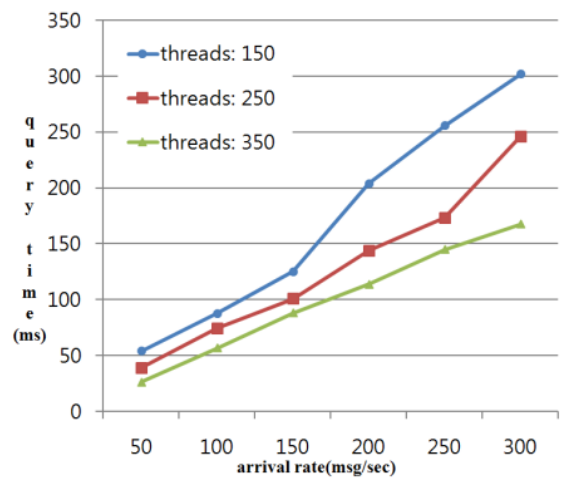

Figure 5. Query Time According to the Number of Threads

\section{Conclusion}

This paper compared simultaneous check-in, fraudulent attendance, prevention of leaving the classroom, and extra equipment according to attendance check-in methods. It also presented a method of app and web-based attendance management to prevent fraudulent attendance. By developing a lab model for OAAS, an experiment was carried out in the classroom and issues and resolution methods were identified. Students preferred BLE-based systems to NFC-based ones since they could check in from their seats. Since web-based attendance check-in requires location information to prevent fraudulent checkin, additional settings were required compared to app-based check-in. RESTful web service-based ROAA-API was defined for attendance management that could be applied to various models of smart devices. For the ROAA-API, performance analysis was conducted according to the types of XML and JSON parsers, and the number of threads. It was assumed that 10,000 students would be making requests for check-in at the same time over the course of 30 seconds. To implement an OAA system for a large number of students' attendance management, it is shown that JSON-based ROAA-API and about 250-350 threads will be required.

As a follow-up, network security function for the ROAA-API in order to protect information exchanged between the OAA-server and client should be added, and the processing overhead from the additional function will be analyzed. 


\section{Acknowledgment}

This work (C0185025) was supported by Business for Cooperative R\&D between Industry, Academy, and Research Institute funded Korea Small and Medium Business Administration in 2015. This work was also supported by a grant from 2015 Research Funds of Andong National University.

\section{References}

[1] D. S. Cho, "An Implementation of Attendance Management System using NFC", Journal of KIICE, vol. 17, no. 7, (2013), pp. 1639-1644.

[2] D. W. Shi, "Automatic Attendance Check System using WiFi Signals based on Smartphone", Journal of KIISE, vol. 19, no. 4, (2013), pp. 219-223.

[3] T. S. Ham, "Smart Phone-Serial Authentication based the Attendance Management System for the Prevention of Proxy Attendance", KIIT Summer Conference, (2013).

[4] H. J. Lee, "Barcode-based Low-cost Portable Training Attendance Management System Development" , JKIECS, vol. 8, no. 11, (2013), pp.1733-1740.

[5] S. J. Jang, "Trend of Technology of NFC", NIPA's weekly Technical Trends, no. 1656, (2014), pp. 1-9.

[6] J. C. Yim, "Tecnology Trends on Proximity Services", ETRI's Electronics and Telecommunication Trend, vol. 30, no. 1, (2015), pp. 123-132.

[7] HTML5 Geolocation, http://www.w3scho ols.com/html/html5_geolocation.asp.

[8] Y. M. Park, "SOAP-based Web Services vs. RESTful Web Services", Electronics and Telecommunications Trends, vol. 25, no. 2, (2010), pp. 112-120.

[9] N. Nurseitov, "Comparison of JSON and XML Data Interchange Formats: A Case Study", CAINE Conference, (2009).

[10] J. S. Oh, "Transmission performance of improvements in mobile applications via XML and JSON data translation", KCC, vol. 39, no. 1, (2012), pp. 129-131.

[11] M. S. Yoo, "Implementation of a Hybrid-App of a Path Tracking System Using Geolocation and Maps on Mobile Devices" , Journal of KONI, vol. 17, no. 6, (2013), pp. 633-637.

[12] S. M. Chun, "Localization of WLAN Access Point Smart Phone's Localization of WLAN Access Point Smart Phone's” , Journal of KICS, vol. 36, no. 2A, (2011), pp. 1-8.

[13] I. S. Choi, "Design and implementation of integrated management system for gas station using web service and sensor network", Journal of KIIT, vol. 10, no. 9, (2012), pp. 105-115.

[14] RESTful URI design, http://blog.2partsmagic.com/restful-uri-design/.

[15] J. M. Apache, “Apache Software Foundation”, http://jmeter.apache.org/.

\section{Authors}

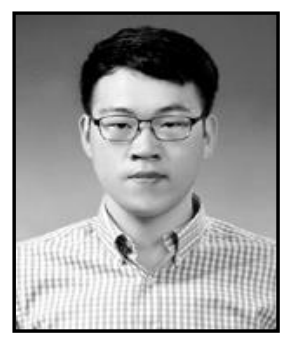

Sun YuXiang, he is now a Masteral course student at the Computer Engineering Department of Andong National University in South Korea. His research interests include network management, RESTful web service, and information modeling.

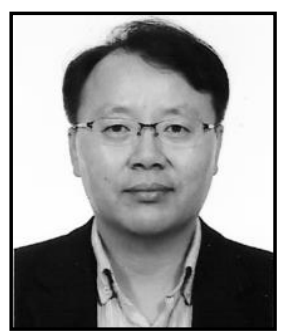

YoungWook Cha (Corresponding Author), he obtained his $\mathrm{PhD}$ in Computer Engineering from the KyungPook National University in South Korea in 1998. He worked for the Electronics and Telecommunications Research Institute as a senior researcher. $\mathrm{He}$ is currently a professor of Computer Engineering Department at Andong National University. His research interests include network management, NGN, security, and network QoS control. 


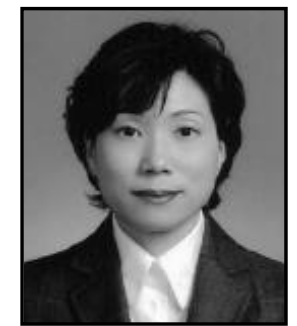

ChoonHee Kim, She obtained her $\mathrm{PhD}$ in Computer Engineering from the KyungPook National University in South Korea in 2000. She worked for ETRI as a researcher. She is currently a professor of the Electronic \& Information Communication Engineering Department at Daegu Cyber University. Her research interests include network management, sensor network, and network QoS control. 
International Journal of $u-$ and e- Service, Science and Technology Vol.8, No. 12 (2015) 